\title{
INCIDENCIA DE LOS CONOCIMIENTOS DE LA ASIGNATURA "CONTABILIDAD PÚBLICA” EN EL DESEMPEÑO PROFESIONAL DE LOS EGRESADOS, EN EL ÁMBITO DE LA ORGANIZACIÓN, GESTIÓN Y CONTROL GUBERNAMENTAL
}

\author{
DOMINGO LUCIANO OVEJERO
}

FACULTAD DE CIENCIAS ECONÓMICAS - UNNE 


\title{
RESUMEN
}

l propósito de esta investigación es analizar si los conocimientos brindados en la
asignatura contabilidad pública resultan suficientes a los profesionales contado-
res, egresados de nuestra Facultad de Ciencias Económicas, para su desempeño
de funciones, tareas o actividades llevadas a cabo en cargos que ocupan en el sector público provincial.

El trabajo permitió identificar, a partir de entrevistas y encuestas semi-estructuradas, el nombre de la jurisdicción, entidad y/o empresa o sociedad del estado en la que se desempeña, el sector al que pertenece ya sea financiero o no financiero, discriminado por cantidad de profesionales por cargo, tarea que realiza, antigüedad y matriculación en el consejo profesional.

Los principales resultados del estudio indicaron los temas que mayor utilidad tienen para el desempeño profesional en las tareas o funciones de los graduados, la aplicación de los conocimientos así como las áreas temáticas vinculadas con las necesidades no cubiertas en la formación de grado en contabilidad pública.

A partir de estos resultados, se realizaran propuestas de modificación del programa de la asignatura, adecuándolo a las necesidades para el desarrollo profesional del contador.

Palabras Claves: contabilidad pública - sector público provincial - egresados contadores - aprendizaje significativo - pertinencia curricular.

\begin{abstract}
The aim of this essay is to analyze the knowledge's application about public accounting by the facultad de ciencias economicas accountant's graduates in their function, tasks or activities at chaco's public sector.

This study has allowed to identify -by using interviews and semi-structured surveys- the district, bureau and/or state's company where the professionals perform their work, being financial or non financial sector, differentiating by professional position, task assigned, antiquity and their enrollment at the consejo profesional de ciencias economicas del Chaco.

The main results of this study indicates the most useful topics involved at professional's management performance in the graduate's functions or tasks, knowledge application and related topics regarding public accounting's unmet needs in undergraduate education.

Proposals will be made considering the investigation's results attending to revise the content of public accounting and adapt it to the accountant's requirement as a professional.
\end{abstract}

Keywords: public accounting - CHACO's public sector - accountant graduates - meaningful learning - curriculum pertinence. 


\section{INTRODUCCIÓN}

Este modesto trabajo sobre incidencia de los conocimientos de la asignatura “contabilidad pública” en el desempeño profesional de los egresados, en el ámbito de la organización, gestión y control gubernamental, es simplemente una síntesis de una parte de un Trabajo de Investigación sobre: "Determinar la incidencia de los conocimientos de Contabilidad Pública en la gestión gubernamental y en el diseño y ejecución de las políticas públicas", que realizara recientemente, como una tarea más de mis actividades docente en la Facultad de Ciencias Económicas de la Universidad Nacional del Nordeste.

Los objetivos generales que se plantean en esta colaboración son:

1.1. Describir y conceptualizar de manera indubitable la importancia y significación que en la formación del profesional Contador Público egresado de nuestra Facultad, tienen los conocimientos adquiridos en la disciplina "Contabilidad Pública".-

1.2. Determinar el grado de inserción y niveles de desempeño del profesional en Ciencias Económicas, en las tareas de gestión y control en las distintas Jurisdicciones y Entidades que conforman el Sector Público.-

1.3. Por su parte, sus objetivos específicos planteados son: Cuál es el verdadero grado de aplicación y la indiscutible e importantísima utilización de dichos conocimientos en el Ejercicio Profesional en el sector público provincial.

1.4. Predeterminar, dentro del sector público, cuál es su conformación en Jurisdicciones, Entidades y Empresas y Sociedades del Estado de la Provincia del Chaco.

1.5. Conocidas ya todas las Jurisdicciones y Entidades que conforman el Sector Público Provincial, determinar principalmente dentro de cada una de ellas, cuales están destinadas a su "Organización”, cuáles a su "Gestión” y cuales a su "Control”.

1.6. Una vez obtenido lo que establece el punto anterior, se debe efectuar un relevamiento de todo el personal que se desempeña en cada Jurisdicción y en cada Entidad, con indicación precisa de su perfil, tarea, cargo que ocupa, misión que cumple, etc.

1.7. Conseguida la información anterior, el trámite siguiente es el de determinar cuántos son profesionales en Ciencias Económicas; de éstos, cuántos son Contadores; qué tipo de tarea, misión o función cumplen y si lo hacen en áreas destinadas a la organización, gestión o control.

1.8. Después de desagregar los múltiples objetivos, presentados en los puntos previamente señalados, expuestos cronológicamente y cómo síntesis, se pretende lograr mediante una encuesta -cuyo Formulario se acompaña- que contenga un cuestionario integral al que se someterá a todos y cada uno de los profesionales Contadores para que manifiesten, en qué medida, de qué manera o porqué esos conocimientos adquiridos al cursar "Contabilidad Pública" en su último año de la carrera le han servido o sido útiles en su desempeño en el Sector Público Provincial, que es en definitiva el objetivo general al que apunta este Trabajo.

1.9. Lograr determinar si el profesional "se halla matriculado en el Consejo Profesional de Ciencias Económicas del Chaco" ya que no es requisito indispensable para el desempeño de 
cargos en el Sector Público Provincial, pero sí lo es y de muchísima importancia desde el punto de vista de sus obligaciones a las que éticamente debe ceñirse en el ejercicio de su Profesión.

\section{MOTIVOS EN LOS QUE SE FUNDAMENTA ESTE TRABAJO}

Entre los motivos que tengo como fundamento principal para escoger el contenido de la temática a tratar, se hallan los siguientes:

1. El haber tenido el privilegio de que el profesor Titular de la asignatura de Contabilidad Pública en la Facultad de Ciencias Económicas, Comerciales y Políticas de la Universidad Nacional del Litoral en Rosario (Santa Fe), en la que cursara mi carrera de Contador Público fue el prestigioso Contador Alberto Arévalo -Contador Emérito de América- que a su vez fuera autor del texto sobre "Contabilidad de las Haciendas Públicas" por el cual estudié la nombrada Disciplina y el que me transmitiera lo esencial del contenido del Programa de la misma y la importancia que ésta tenía para el Ejercicio Profesional en el ámbito Público.

2. Mi desempeño durante muchísimos años como Director de Administración del Ministerio de Economía y Obras Públicas de la Provincia del Chaco, el que me permitió no solamente aplicar los principios y preceptos de esta disciplina, sino también toda la normativa que rige el accionar de la Hacienda del Estado y de su Administración Pública; es decir que si a la excelencia que caracterizó al profesor que supo transmitirme conocimientos valiosos de esa materia, le agrego la gran experiencia adquirida en el Sector Público Provincial en el que cumpliendo la misión de Jefe de un Servicio Administrativo importantísimo durante 20 años, apliqué dichos conocimientos y los incrementé en grado sumo al desempeñarme posteriormente dentro del mismo Sector Público Provincial, en un periodo de 10 años más, como Director de Capacitación y Normatización del Personal del Gobierno de la Provincia del Chaco, función que me permitió agregar a la gran experiencia adquirida como Jefe de un Servicio Administrativo en una muy importante tarea de "gestión ejecutiva”, una misión -para mí añorada- cual era la de poder participar en forma personal y directa en la Organización de Cursos de Capacitación para el Personal de la Administración Pública Provincial, en el Diseño, Contenido Programático y pautas a las que se debieron ajustar los mismos, como así también el Perfil que se fijó para quienes asistieron en su condición de alumnos, como de los docentes que tuvieron a cargo su dictado, ya que esa experiencia en el ejercicio de esa función, me permitió "detectar" las insuficiencias y deficiencias que devienen de las propias carencias que en nuestro accionar cotidiano como agentes o empleados de Estado se ponen de manifiesto, pues en ellas justamente encontré los Temas que debían incluir los “Temarios o Programas a Desarrollar” vinculados con: a) La Organización, b) La Gestión y c) El Control que son los que sirvieron de base para muchos de esos cursos que con mucha asistencia y mucho éxito se dictaron durante el lapso en que me desempeñara en dicho cargo; porque además de involucrarme en su organización, también tuve injerencia en el dictado de muchas clases de aquellos que sus Programas contenían temas de la Contabilidad Pública. 
Intervinieron en éstos, principalmente, agentes y funcionarios de los Servicios Administrativos de todas las Jurisdicciones y Entidades; de la Contaduría General; del Tribunal de Cuentas; de la Fiscalía de Estado; de la Fiscalía de Investigaciones Administrativas, entre otras.-

Debo agregar a ello también como "motivante" el haber participado de innumerables Reuniones, Congresos y Jornadas en representación de la Cátedra y/o del Estado Provincial, en las que se trataran temas vinculados con nuestra materia, y en las que integré siempre las Comisiones relacionadas con el Sector Público, organizadas por el Consejo Profesional de Ciencias Económicas o por el Colegio de Graduados en Ciencias Económicas de Buenos Aires y a partir de la creación de nuestra Asociación argentina de Profesores Universitarios de Contabilidad Pública - de cuyo consejo directivo forme parte desde su creación, integrando últimamente su comité académico de honor- y de la Organización de los XXVIII simposios realizados hasta la fecha , a los que siempre asistí y en los que se trataron extensos temarios sobre asuntos que a la par de considerar cuestiones importantes de la Materia y preocupaciones que se reflejaran en sus conclusiones y a su vez sirvieran muchas de ellas como recomendaciones que tiendan todas a un mejoramiento no sólo en lo académico docente, sino también en propuestas que se traduzcan en Reformas Substanciales en la Legislación, que traigan consigo un Accionar Gubernamental transparente, eficaz y eficiente cuyo logro final sea el Bienestar General de la Población y para ello nada mejor que tener presente, además del concepto de contabilidad pública fundamentalmente el objeto de la misma, al que el Primer Simposio Nacional de Profesores Universitarios de Contabilidad Pública celebrado en la ciudad de Bahía Blanca en mayo de 1981 lo definió como "El Estudio de la Hacienda Pública desde los puntos de vista de su: a) organización; b) gestión; y c) control”, elementos sustanciales y basamento fundacional de este trabajo.

Estos simposios, organizados año tras año por las cátedras de Contabilidad Pública y la Asociación Argentina de Profesores Universitarios de Contabilidad Pública en las distintas Facultades de Ciencias Económicas de todas las Universidades Nacionales del país, incluyendo también a la Universidad Católica, me sirvieron también para ir conociendo en profundidad a todos y cada uno de sus integrantes, excelentes docentes entre los que se hallan también prestigiosos autores de textos de estudio; obras y/o trabajos meritorios referidos íntegramente a la Contabilidad Pública, a su estudio o relacionados con la misma, que además de resultarme atractivos, sus contenidos me sirvieron para repasar, confirmar y también adquirir nuevos conocimientos y, fundamentalmente, para estar siempre actualizado en relación a los mismos, los que además de utilizarlos para mi consulta personal, conforman parte de la bibliografía de la materia, algunos como el del Profesor Atchabahian desde el año 1963. En ellos, nuestros alumnos encuentran valiosa información que no solo les resulta indispensable para el estudio de esta disciplina, sino también para su formación profesional, que seguramente les será de mucha utilidad a aquellos que se desempeñen luego en el Sector Público y, en gran medida, para beneficio de la comunidad toda.

Todo lo expuesto en relación a mi actividad en el Sector Público Provincial contribuyó para que en el ejercicio de la docencia universitaria en la Facultad de Ciencias Económicas de la Uni- 
versidad Nacional del Nordeste en la carrera de Contador Público, en la Asignatura Contabilidad Pública como Profesor Titular desde hace 53 años ininterrumpidamente, en cuyo desarrollo y al dictar cada una de las unidades que integran su programa, haya intentado por todos los medios a mi alcance de hacerles ver a mis alumnos la importancia que la misma tiene en la Currícula.

\section{RESULTADO FINAL}

Después de lo que señalo en los puntos anteriores, en los que efectúo un breve relato de mi desempeño en el sector público provincial y en la docencia universitaria como Profesor Titular de la Asignatura Contabilidad Pública, en ambas actividades durante muchísimos años. Es mi deber moral aclarar que mi intención no es -de ninguna manera- pretender hacer conocer parte de mi curriculum vitae, es simplemente al solo efecto de demostrar con hechos y en forma indubitable que la tarea que me propuse con este trabajo sobre Incidencia de los conocimientos de la asignatura "Contabilidad Pública" en el desempeño profesional de los egresados, en el ámbito de la organización, gestión y control gubernamental es absolutamente factible, real y cierta.

\section{CONCLUSIONES}

De la lectura de las Conclusiones surge sin duda alguna que el resultado final obtenido fue el esperado, ya que los hechos así lo han demostrado en:

4.1. Las reuniones realizadas con los profesionales Contadores que tienen jerarquía intermedia (Jefe de Sección a Jefes de Departamento) y Directores o que conforman el núcleo de Autoridades Superiores (Ministros, Secretarios de Estado, Directores de Empresas y Sociedades del Estado, etc.).

4.2. Que se logró obtener la conformación de la Estructura del Sector Público Provincial en Jurisdicciones, Entidades y Empresas y Sociedades del Estado Provincial (ver anexo A).

4.3. La obtención de los instrumentos legales indispensables, como: La Constitución Provincial, la Ley 4787 de Organización y Funcionamiento de la Administración Financiera del Sector Público de la Provincia del Chaco, la Ley Orgánica del Tribunal de Cuentas de la Provincia del Chaco y su Reglamentación, la Ley de Obras Públicas, la Ley de Ministerios, el Régimen de Contrataciones para la Adjudicación de Suministros, Construcción de Obras y Concesión de Obras Públicas y/o Servicios.-

4.4. Conocidas ya todas las Jurisdicciones y Entidades, así como sus propias estructuras y los Servicios Públicos Indivisibles que prestan, resultó fácil determinar en cada una de ellas su Organización, así como el tipo de tareas que en ellas se realizan, la naturaleza de las mismas y si las acciones se caracterizan por ser de gestión, de control o de otra índole; por ejemplo, sabemos que la Contaduría General es el órgano de control interno y que sus contadores auditores realizan tareas de control o auditoría; que el Tribunal de Cuentas es el órgano de control exter- 
no y que sus contadores fiscales cumplen funciones de fiscalización y control; en cambio, un Servicio Administrativo es un órgano de gestión ejecutiva y las funciones que cumplen muchos de los contadores que se desempeñan en él son, fundamentalmente, de gestión.-

4.5. Para saber la cantidad de personas que se desempeñan en cada Jurisdicción y Entidad como así también su perfil, tarea, cargo que ocupan y misión que cumplen, utilicé el Censo del Personal que me lo suministró el Área pertinente.

4.6. De la información lograda obtuve la nómina de contadores y la jurisdicción, entidad y empresa o sociedad del estado en la que se desempeña cada uno.

4.7. A posteriori, se procedió a la entrega del formulario de encuesta -como el que se adjuntaconjuntamente con el Programa oficial de la materia -el que después de ser llenado por cada Contador, fue retirado y analizado exhaustivamente-, lo que me sirvió para confeccionar la planilla que como anexo A - sector público de la Provincia del Chaco, que se agrega a este trabajo como parte integrante del mismo y es al que debe remitirse para obtener la siguiente información:

4.7.1. Nómina de las jurisdicciones, las entidades y las empresas y sociedades del Estado provincial.

4.7.2. Cuántos Contadores se desempeñan en cada una de ellas.

4.7.3. Qué cargo ocupan.

4.7.4. Qué Tareas o Funciones desempeñan.

4.7.5. Cuál es su Antigüedad.

4.7.6. Otro dato obtenido de las encuestas, suministrado por cada Contador encuestado, es el relacionado con la inscripción en la Matrícula en el Consejo Profesional de Ciencias Económicas del Chaco, el que también consideré siempre relevante porque está vinculado con el ejercicio de la profesión en el Sector Público.-

4.7.8. Por último, la información más importante que mediante la encuesta obtuve de cada uno de los profesionales Contadores que realizan tareas en el sector público provincial es la respuesta a la pregunta. "De los conocimientos adquiridos al cursar la Asignatura Contabilidad Pública y referido a los contenidos del programa, ¿Cuáles -según su opinión- le fueron de mayor utilidad en la función, trabajo o tarea que Ud. realiza actualmente o realizó con anterioridad?”

¿Por qué considero que es la más importante?: $1^{\circ}$ ) Porque en las respuestas dadas por cada uno de los encuestados se halla la razón de ser o dicho de otra manera el leitmotiv de este trabajo; $2^{0}$ ) Por el contenido de dichas respuestas; algunas sintéticas, otras muy extensas, pero todas de gran valor y trascendencia, que demuestran inequívocamente que vale la pena que sigamos nutriéndonos de su esencia, jerarquizándola como la mejor forma de jerarquizarnos, homenajeando a sus maestros y transmitiendo a nuestros alumnos lo esencial de su contenido. 


\section{ANEXO A - SECTOR PUBLICO DE LA PROVINCIA DEL CHACO}

\begin{tabular}{|c|c|c|c|c|c|c|c|c|c|c|c|}
\hline \multirow{2}{*}{$\begin{array}{l}\text { NO FINANCIERO } \\
\text { JURISDICCIONES }\end{array}$} & \multirow[b]{2}{*}{$\begin{array}{l}\text { TOTAL } \\
\text { GRAL. }\end{array}$} & \multicolumn{3}{|c|}{$\begin{array}{l}\text { CARGO QUE } \\
\text { OCUPA }\end{array}$} & \multicolumn{5}{|c|}{$\begin{array}{l}\text { TAREAS O FUNCIONES } \\
\text { QUE DESEMPEÑAN }\end{array}$} & \multicolumn{2}{|c|}{$\begin{array}{l}\text { ANTIGÜE- } \\
\text { DAD }\end{array}$} \\
\hline & & $\begin{array}{l}\text { AUT. } \\
\text { SUPER. }\end{array}$ & $\begin{array}{l}\text { DIREC } \\
\text { TORES }\end{array}$ & $\begin{array}{l}\text { JEFE } \\
\text { SEC } \\
\text { DIV } \\
\text { DPTO }\end{array}$ & $\begin{array}{l}\text { GES- } \\
\text { TION }\end{array}$ & $\begin{array}{l}\text { AUDI- } \\
\text { TORIA }\end{array}$ & $\begin{array}{l}\text { CON- } \\
\text { TROL }\end{array}$ & $\begin{array}{l}\text { FISCA- } \\
\text { LIZAC. }\end{array}$ & $\begin{array}{l}\text { ASE- } \\
\text { SOR }\end{array}$ & $\begin{array}{l}\text { - DE } 5 \\
\text { AÑOS }\end{array}$ & $\begin{array}{l}+ \text { DE } 5 \\
\text { AÑOS }\end{array}$ \\
\hline $\begin{array}{c}\text { PODER } \\
\text { LEGISLATIVO }\end{array}$ & 22 & 02 & 02 & 18 & 14 & 01 & 02 & 01 & 04 & 07 & 15 \\
\hline $\begin{array}{l}\text { PODER } \\
\text { JUDICIAL }\end{array}$ & 43 & - & 03 & 40 & 26 & 05 & 05 & 04 & 02 & 14 & 29 \\
\hline GOBERNACION & 13 & 03 & 02 & 08 & 09 & 01 & 01 & 01 & 01 & 07 & 06 \\
\hline $\begin{array}{l}\text { ADMIN. } \\
\text { TRIBUT. PROV. }\end{array}$ & 72 & - & 02 & 70 & 20 & 06 & 06 & 20 & 20 & 32 & 40 \\
\hline $\begin{array}{l}\text { MRIO. DE LA } \\
\text { PRODUC. }\end{array}$ & 28 & - & 02 & 26 & 12 & 03 & 10 & 02 & 01 & 18 & 10 \\
\hline $\begin{array}{l}\text { MRIO. SALUD } \\
\text { PUBLICA }\end{array}$ & 19 & - & 02 & 17 & 09 & 02 & 07 & - & 01 & 10 & 09 \\
\hline $\begin{array}{l}\text { MRIO. ECON. } \\
\text { O. Y S. PUB }\end{array}$ & 15 & 02 & 02 & 11 & 06 & 02 & 03 & 01 & 03 & 06 & 09 \\
\hline $\begin{array}{l}\text { MRIO. GOB. } \\
\text { JUS. Y TRAB }\end{array}$ & 12 & - & 02 & 10 & 04 & 01 & 01 & 06 & - & 03 & 09 \\
\hline $\begin{array}{l}\text { MRIO. EDUC } \\
\text { C. Y TECN. }\end{array}$ & 20 & - & 02 & 18 & 14 & 02 & 02 & 01 & 01 & 10 & 10 \\
\hline $\begin{array}{l}\text { MRIO. DESARR. } \\
\text { SOCIAL }\end{array}$ & 22 & - & 02 & 20 & 10 & 03 & 03 & 04 & 02 & 17 & 05 \\
\hline $\begin{array}{l}\text { SEC.PLAN. } \\
\text { Y EVAL. RES. }\end{array}$ & 05 & - & 01 & 04 & 02 & - & 01 & - & 02 & 03 & 02 \\
\hline $\begin{array}{l}\text { SEC. TRANS. } \\
\text { O. Y S. P. }\end{array}$ & 04 & - & 01 & 03 & 02 & - & 01 & 01 & - & 01 & 03 \\
\hline $\begin{array}{l}\text { SEC. REP Y } \\
\text { PROM PROV }\end{array}$ & 04 & - & - & 04 & 04 & - & - & - & - & 02 & 02 \\
\hline $\begin{array}{l}\text { CONTADURIA } \\
\text { GRAL. }\end{array}$ & 35 & 02 & 04 & 29 & 05 & 14 & 12 & 03 & 01 & 06 & 29 \\
\hline $\begin{array}{l}\text { TESORERIA } \\
\text { GRAL. }\end{array}$ & 09 & 01 & 02 & 06 & 03 & 03 & 02 & 01 & - & 02 & 07 \\
\hline $\begin{array}{l}\text { TRIBUNAL DE } \\
\text { CUENTAS }\end{array}$ & 81 & 05 & 07 & 69 & 06 & 15 & 25 & 35 & - & 33 & 48 \\
\hline $\begin{array}{l}\text { FISCALIA DE } \\
\text { ESTADO }\end{array}$ & 02 & - & - & 02 & - & - & - & 01 & 01 & - & 02 \\
\hline $\begin{array}{l}\text { FISCALIA INV. } \\
\text { ADMIN. }\end{array}$ & 04 & - & 01 & 03 & - & 01 & 01 & 01 & 01 & - & 04 \\
\hline \multirow[t]{2}{*}{$\begin{array}{c}\text { POLICIA } \\
\text { PROVINCIAL }\end{array}$} & 06 & - & - & 06 & 02 & 01 & 02 & 01 & - & 03 & 03 \\
\hline & 416 & 15 & 37 & 364 & 148 & 61 & 84 & 83 & 40 & 174 & 242 \\
\hline
\end{tabular}




\begin{tabular}{|c|c|c|c|c|c|c|c|c|c|c|c|}
\hline \multirow{2}{*}{$\begin{array}{c}\text { NO FINANCIERO } \\
\\
\text { ENTIDADES } \\
\text { ESPECIALES }\end{array}$} & \multirow[b]{2}{*}{$\begin{array}{l}\text { TOTAL } \\
\text { GRAL. }\end{array}$} & \multicolumn{3}{|c|}{$\begin{array}{l}\text { CARGO QUE } \\
\text { OCUPA }\end{array}$} & \multicolumn{5}{|c|}{$\begin{array}{l}\text { TAREAS O FUNCIONES } \\
\text { QUE DESEMPEÑAN }\end{array}$} & \multicolumn{2}{|c|}{$\begin{array}{l}\text { ANTIGÜE- } \\
\text { DAD }\end{array}$} \\
\hline & & $\begin{array}{l} \\
\text { AUT. } \\
\text { SUPER. }\end{array}$ & $\begin{array}{l}\text { DIREC } \\
\text { TORES }\end{array}$ & \begin{tabular}{|c} 
JEFE \\
SEC \\
DIV \\
DPTO
\end{tabular} & $\begin{array}{l}\text { GES- } \\
\text { TION }\end{array}$ & $\begin{array}{l}\text { AUDI- } \\
\text { TORIA }\end{array}$ & $\begin{array}{l}\text { CON- } \\
\text { TROL }\end{array}$ & $\begin{array}{l}\text { FISCA- } \\
\text { LIZAC. }\end{array}$ & $\begin{array}{l}\text { ASE- } \\
\text { SOR }\end{array}$ & $\begin{array}{l}- \text { DE } 5 \\
\text { AÑOS }\end{array}$ & $\begin{array}{l}+ \text { DE } 5 \\
\text { AÑOS }\end{array}$ \\
\hline $\begin{array}{l}\text { LOTERIA } \\
\text { CHAQUEÑA }\end{array}$ & 08 & - & 04 & 04 & 04 & 01 & 01 & 01 & 01 & - & 08 \\
\hline \multirow{2}{*}{$\begin{array}{l}\text { I.N.S.S.E. Y } \\
\text { PRESTAMOS }\end{array}$} & 30 & 01 & 05 & 24 & 14 & 05 & 05 & 02 & 03 & 15 & 15 \\
\hline & 38 & 01 & 09 & 28 & 19 & 06 & 06 & 03 & 04 & 15 & 23 \\
\hline \multicolumn{12}{|l|}{$\begin{array}{l}\text { EMPRESAS } \\
\text { Y SOC. }\end{array}$} \\
\hline S.A.M.E.E.P. & 09 & 01 & 02 & 06 & 03 & 02 & 02 & 02 & - & 02 & 07 \\
\hline S.E.C.H.E.E.P. & 11 & - & 04 & 07 & 05 & 02 & 02 & 01 & 01 & - & 11 \\
\hline SEFECHA & 01 & - & - & 01 & 01 & - & - & - & - & 01 & - \\
\hline $\begin{array}{c}\text { ECOM- } \\
\text { CHACO S.A. }\end{array}$ & 04 & - & 02 & 02 & 01 & 01 & 01 & 01 & - & - & 04 \\
\hline \multirow{2}{*}{$\begin{array}{l}\text { FIDUC. DEL } \\
\text { NORTE S.A. }\end{array}$} & 08 & 02 & 02 & 04 & 04 & 01 & 01 & 01 & 01 & - & 08 \\
\hline & 33 & 03 & 10 & 20 & 14 & 06 & 06 & 05 & 02 & 03 & 30 \\
\hline \multicolumn{12}{|l|}{ FINANCIERO } \\
\hline \multirow[t]{2}{*}{$\begin{array}{c}\text { NUEVO BANCO } \\
\text { CHACO }\end{array}$} & 77 & 04 & 08 & 65 & 47 & 09 & 06 & 10 & 05 & 32 & 45 \\
\hline & 591 & 24 & 69 & 498 & 246 & 84 & 106 & 103 & 52 & 239 & 352 \\
\hline
\end{tabular}

\begin{tabular}{|c|c|c|}
\hline \multicolumn{3}{|l|}{ RESUMEN } \\
\hline \multicolumn{3}{|l|}{ SECTOR PUBLICO NO FINANCIERO } \\
\hline JURISDICCIONES & 416 & \\
\hline ENTIDADES & 27 & \\
\hline ENTIDADES ESPECIALES & 38 & \\
\hline EMPRESAS Y SOC. DEL ESTADO & 33 & 514 \\
\hline \multicolumn{3}{|l|}{ SECTOR PUBLICO FINANCIERO } \\
\hline NUEVO BANCO DEL CHACO & 77 & 77 \\
\hline TOTAL GENERAL & & 591 \\
\hline
\end{tabular}




\section{INFORMACIÓN COMPLEMENTARIA}

Contadores Públicos Egresados en los últimos 30 años de la UNNE 4503.

Contadores Públicos Egresados en los últimos 30 años Matriculados en Consejo Profesional de Ciencias Económicas del Chaco 2112 - 47\%.

Contadores Públicos Egresados que cumplen funciones en Sector Público Provincial 591 - 13\%.

Contadores Públicos Egresados con funciones en el Sector Público Matriculados 100 - 17\%.

\section{FORMULARIO ENCUESTA

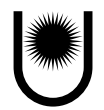 \\ Universidad. Nacional del. Nordeste \\ Facultad de Giencias Ëconómicas}

\section{CATEDRA: CONTABILIDAD PÚBLICA}

- Encuesta dirigido a los Contadores Públicos del Sector Público Provincial

Objetivo:

Contribuir con su valiosa opinión para la concreción del trabajo de Investigación: "Grado de aplicación de los conocimientos impartidos al cursar "Contabilidad Pública", en las funciones que realicen nuestros egresados en el sector público“.
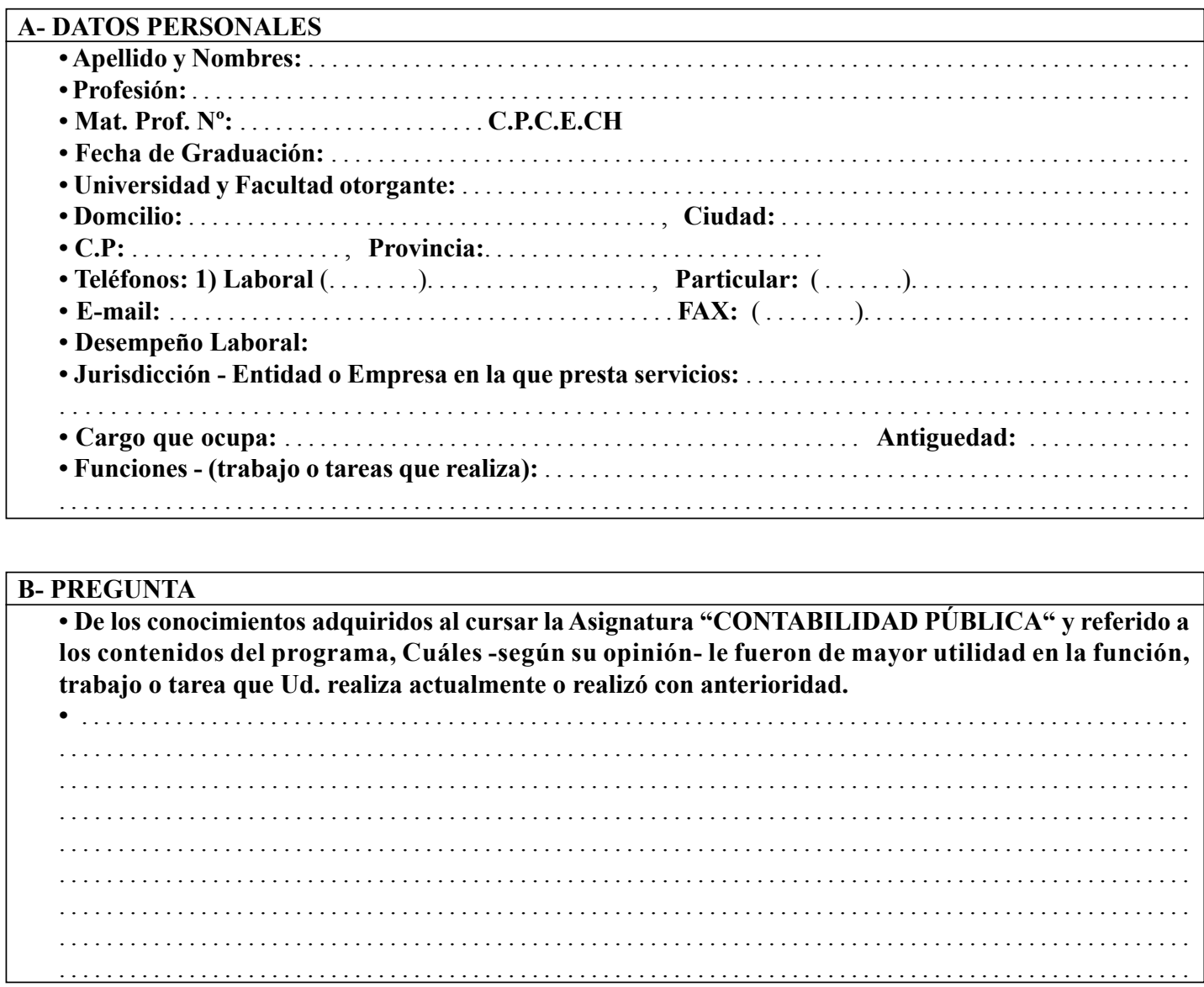


\section{CURRÍCULUMM VITAE}

DOMINGO LUCIANO OVEJERO.

Contador público y perito partidor (Universidad Nacional del Litoral).

Profesor titular de Contabilidad Pública, en la Facultad de Ciencias Económicas de la Universidad Nacional del Nordeste.

Miembro del comité académico de honor de la Asociación Argentina de Profesores Universitarios de Contabilidad Pública.

minachon@arnet.com.ar 